\title{
Ginsenoside Rg1 Protects against Non-alcoholic Fatty Liver Disease by Ameliorating Lipid Peroxidation, Endoplasmic Reticulum Stress, and Inflammasome Activation
}

\author{
Yashu Xu, Cheng Yang, Shujun Zhang, Jiajun Li, Qing Xiao, and Wenxiang Huang* \\ Department of Infectious Diseases, The First Affiliated Hospital of Chongqing Medical University; Chongqing \\ 400016, China. \\ Received February 18, 2018; accepted August 3, 2018; advance publication released online August 21, 2018
}

\begin{abstract}
Non-alcoholic fatty liver disease (NAFLD) is increasingly prevalent and represents a growing challenge in terms of prevention and treatment. The purpose of this study is to investigate the protective effects of ginsenoside Rg1 (Rg1), an active ingredient of a natural medicine, and further clarify its protective mechanisms, in a mouse model of NAFLD induced by a high-fat diet. Rg1 significantly reduced liver weight, serum alanine aminotransferase (ALT), aspartate aminotransferase (AST), triglyceride (TG), liver free fatty acids (FFAs) and malondialdehyde (MDA) levels, and increased superoxide dismutase (SOD) activity. Rg1 also upregulated the expression of peroxisome proliferator-activated receptor-alpha (PPAR $\alpha$ ), which stimulated fatty acid beta oxidation and promoted the metabolism of FFAs and TG. It also suppressed the expression of CCAAT/enhancer binding protein (C/EBP) homologous protein (CHOP), cysteine-containing aspartatespecific proteases 12 (Caspase 12), and glucose-regulated protein78 (GRP78), which reduced endoplasmic reticulum (ER) stress. Furthermore, Rg1 alleviated liver inflammation by inhibiting the activation of nucleotide binding oligomerization domain (NOD)-like receptor family pyrin domain-containing 3 (NLRP3) and thus reduced the production of inflammatory cytokines, such as interleukin 1-beta (IL-1 $\beta$ ) and interleukin 18 (IL-18). These results suggested that Rg1 may protect against NAFLD, through regulation of lipid peroxidation, ER stress and inflammasome activation.
\end{abstract}

Key words ginsenoside Rg1; non-alcoholic fatty liver disease; lipid peroxidation; endoplasmic reticulum stress

Non-alcoholic fatty liver disease (NAFLD) is the hepatic manifestation of the metabolic syndrome that is not caused by alcohol consumption. ${ }^{1)}$ NAFLD has raised significant burdens to human health by affecting approximately a quarter of the world's population. $^{2,3)}$ The primary clinical manifestation of NAFLD could be non-alcoholic fatty liver disease (NAFL) and non-alcoholic fatty hepatitis (NASH), which may transform into liver fibrosis, cirrhosis and hepatocellular carcinoma at later stages. ${ }^{4,5)}$ NAFLD can also increase the risk of cardiovascular, cancer and liver-related deaths. ${ }^{6}$ Although lifestyle changes and exercise can delay the development of NAFLD, the combination of appropriate drugs is recommended for the treatment of this disease. To date, no pharmacological treatment is specifically designed for NAFLD. ${ }^{7)}$ Thus, identifying drugs that can effectively block the progression of NAFLD are needed.

The pathogenesis of NAFLD is complicated and multifactorial. In the liver, free fatty acids (FFAs) are consumed by $\beta$-oxidation and stored as triglycerides in lipids. The accumulation of FFAs will induce mitochondrial dysfunction, endoplasmic reticulum (ER) stress and the development of lipotoxicity. ${ }^{8,9)}$ Peroxisome proliferator-activated receptoralpha (PPAR $\alpha$ ), which can be activated by fatty acids as well as other peroxisome proliferators, plays a critical role in the $\beta$-oxidation of FFAs and subsequently protects against dyslipidemia. ${ }^{10)}$ The ER stress response, on the other hand, has been linked to obesity, diabetes and liver cancer through induction of inflammation, apoptosis and insulin resistance. ${ }^{11,12)}$ ER stress may be also involved in the progression of NAFLD, as evidenced by the observations that NASH patients expressed elevated levels of ER stress markers, including CCAAT/enhancer binding protein $(\mathrm{C} / \mathrm{EBP})$ homologous protein $(\mathrm{CHOP})$, glucose-regulated protein78 (GRP78) and cysteine-containing aspartate-specific proteases 12 (Caspase 12). ${ }^{13-15)}$ More recently, it was shown that ER stress induced inflammation and cell death associated with the activation of nucleotide binding oligomerization domain (NOD)-like receptor family pyrin domain-containing 3 (NLRP3) inflammasome, which is a multi-protein complex that can sense obesity-associated danger signals, FFAs, and mediate the maturation of pro-inflammatory cytokines interleukin- $\beta$ (IL-1 $\beta$ ) and interleukin 18 (IL-18). ${ }^{16,17)}$ Those findings raised the possibility that suppression of ER stress responses as well as NLRP3 inflammasome activation might be beneficial to the treatment of NAFLD.

Natural products derived from plants with medicinal properties have a good potential for curing human diseases. In recent years, an increasing number of studies have focused on herbal extracts or natural products with anti-hyperlipidemia and liver protection against NAFLD. ${ }^{18)}$ Ginseng is a traditional herbal medicine that is widely used in the world, especially in east Asia, reported to possess a good effect of the hypoglycemic activity. ${ }^{19,20)}$ Many studies have also explored the medicinal value of Ginsenoside Rg1 (Rg1) (Fig. 1A), which is one of the most effective monomers extracted from ginseng, in the treatment of liver diseases. ${ }^{21)}$ In fact, our previous data has shown that $\mathrm{Rg} 1$ can reduce liver damage in acute liver failure (ALF) and alcoholic liver disease (ALD), by means of anti-inflammatory and anti-apoptosis effects. ${ }^{22,23)}$ In addition to those data, Rg1 has been shown to improve insulin resistance, which is expected to be a contributing factor to 
A

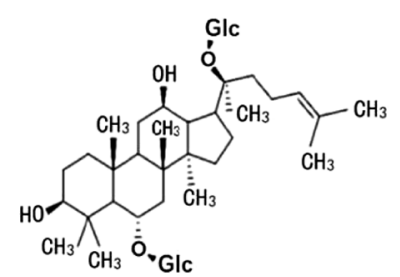

B

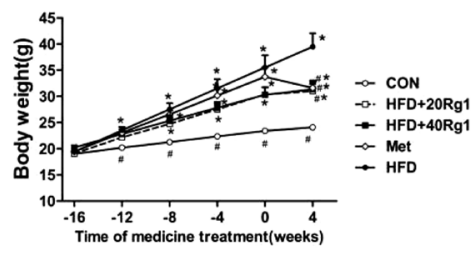

D

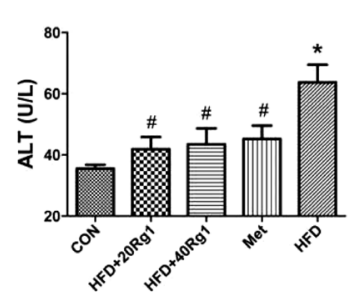

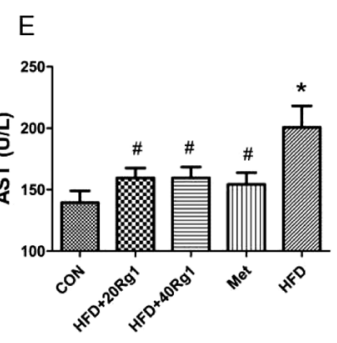

F

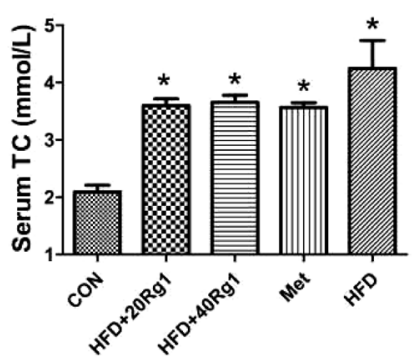

$\mathrm{H}$

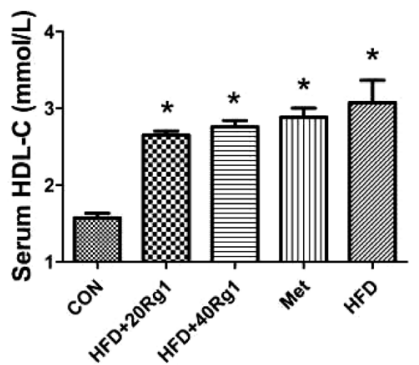

G

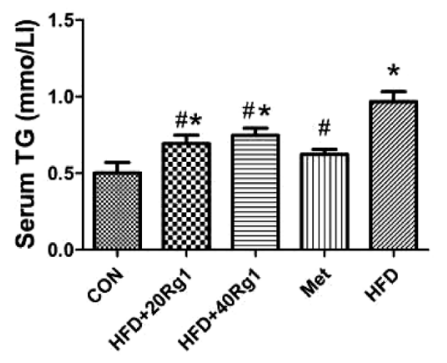

I
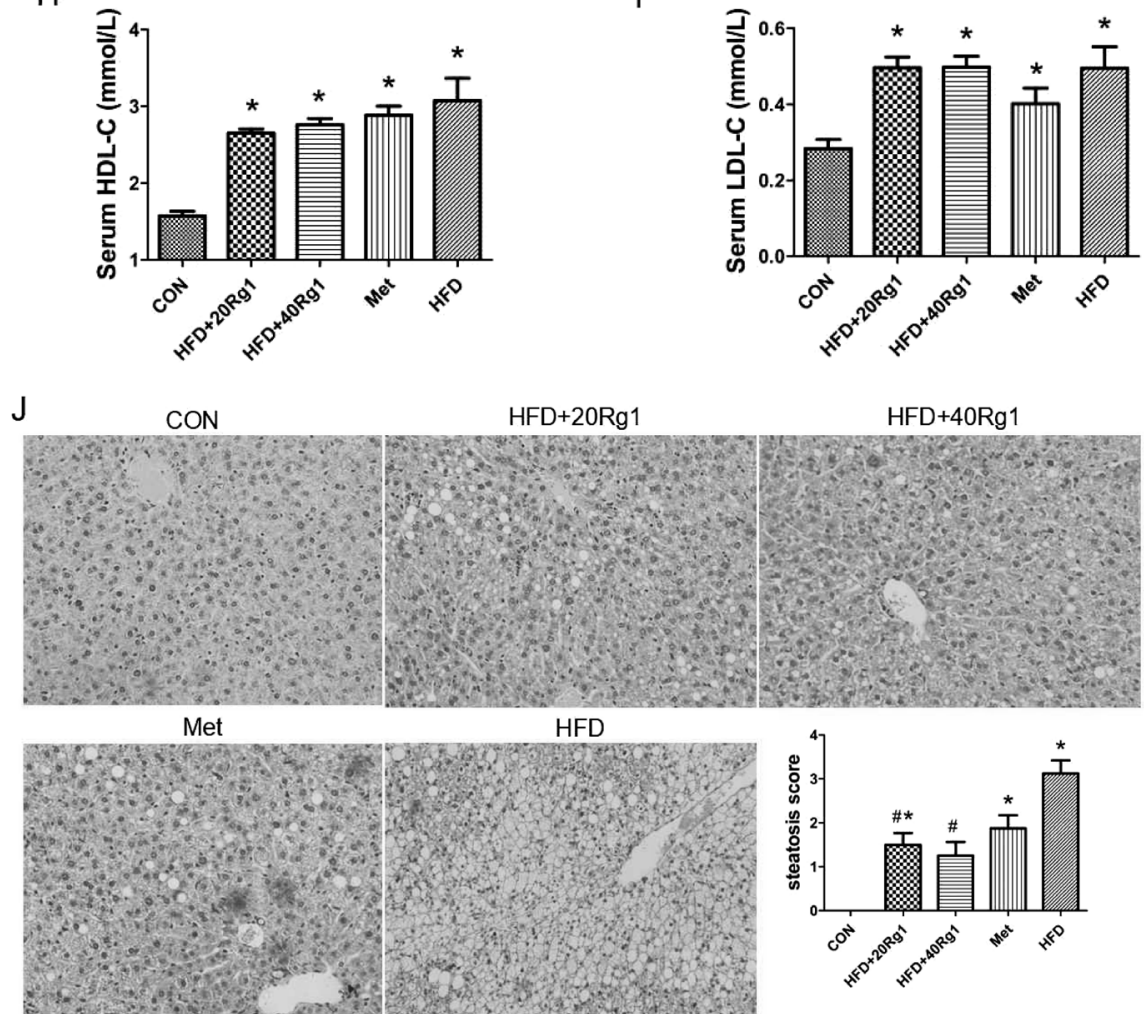

HFD+40Rg1

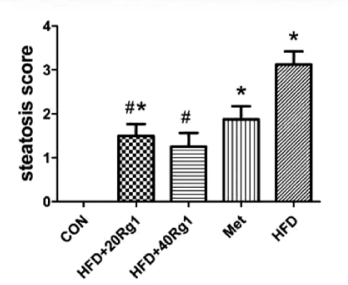

Fig. 1. Hepatoprotection of Rg1 against HFD-Induced Liver Steatosis

Chemical structure of ginsenoside Rg1 (A). Effects of Rg1 on body weight gain (B) and liver wet weight (C) in mice. Serum ALT (D), AST (E), TC (F), TG (G), HDL $(\mathrm{H})$ and LDL (I) levels elevated by HFD were significantly reduced by treatment with both doses of $\mathrm{Rg} 1$. Data are means \pm standard error of the mean $(n=8)$. * $p<0.05$ versus $\mathrm{CON},{ }^{\#} p<0.05$ versus HFD. Rg1 attenuated liver injury induced by HFD in mice. (J) Representative H\&E stained liver sections (400× magnification). The histopathology steatosis score was calculated. CON, control group; HFD+20 Rg1, Rg1 low-dose group; HFD+40 Rg1, Rg1 high-dose group; Met, metformin group; HFD, high-fat diet group. 
NAFLD. ${ }^{24)}$ Based on these studies, we trend to determine whether Rg1 could protect NAFLD as well as the underlying molecular mechanisms in case of therapeutic applications. Our data would provide significant insights for the development of novel treatment strategies for NAFLD.

\section{MATERIALS AND METHODS}

Reagents $\operatorname{Rg} 1$ (>98\% purity) and metformin hydrochloride $(>98.5 \%$ purity) were purchased from Meilun Biotechnology Co., Ltd. (Dalian, China). Malondialdehyde (MDA), superoxide dismutase (SOD) and FFA assay kits were purchased from Nanjing Jiancheng Institute of Biotechnology (Nanjing, China). Antibodies against $\beta$-actin, glyceraldehyde3-phosphate dehydrogenase (GAPDH), GRP78, CHOP and NLRP3 were purchased from Wanlei Biotechnology Co., Ltd. (Shenyang, China). Antibodies against Caspase 12, IL-1 $\beta$, and horseradish peroxide (HRP)-conjugated anti-rabbit immunoglobulin G (IgG), were purchased from Cell Signaling Technology (Beverly, MA, U.S.A.).

Ethics Statement All the following procedures were strictly faithful to $3 \mathrm{R}$ principles, and animal care and housing procedures conformed to Chinese regulatory requirements. The study protocol was approved by the Ethics Committee of Chongqing Medical University (Chongqing, China). All animals received humane care in compliance with the Guide for the Care and Use of Laboratory Animals published by the National Institutes of Health. Special care was taken to minimize number of and suffering of animals.

Animals and Treatments Healthy female C57BL/6 mice were transferred at 6-8 weeks of age to the laboratory animal facilities where they were maintained at a 12-h light/dark cycle and a constant temperature of $26^{\circ} \mathrm{C}$. After 1 week of acclimatization, they were randomly allocated to five experimental groups of eight mice per group. These were control (CON), high-fat diet (HFD), Rg1 low-dose (HFD+20 Rg1), Rg1 highdose (HFD+40 Rg1), and metformin (Met) groups. The CON group was fed with a normal diet (Chongqing Medical University Laboratory Animal Center, Chongqing, China). The other groups were fed with a HFD of $60 \%$ fat, $20 \%$ protein, and 20\% carbohydrate. (Research Diet D12492, New Brunswick, NJ, U.S.A.). All groups had free access to water and food. After 16 weeks of feeding, the CON and HFD animals were given by gavage daily with $0.9 \%$ saline $(20 \mathrm{mg} / \mathrm{kg})$. The HFD+20 Rg1 and HFD+40 Rg1 group animals through administration by gavage daily with $20 \mathrm{mg} / \mathrm{kg}$ and $40 \mathrm{mg} / \mathrm{kg} \mathrm{Rg} 1$ respectively. The Met group animals were given by gavage daily with metformin $(150 \mathrm{mg} / \mathrm{kg})$. Each group was treated for 1 month. The mice were fasted for $12 \mathrm{~h}$ with no water restriction after the completion of treatment. All mice were sacrificed, with blood and liver samples collected for further analysis. The blood samples were centrifuged at $4000 \mathrm{rpm}$ for $15 \mathrm{~min}$ at $4^{\circ} \mathrm{C}$ to obtain the serum. The liver samples were dissected and fixed in $10 \%$ formalin for histological analysis or snap frozen stored at $-80^{\circ} \mathrm{C}$.

Biochemical Assays Serum alanine aminotransferase (ALT), aspartate aminotransferase (AST), triglycerides (TG), total cholesterol (TC), high-density lipoprotein cholesterol (HDL-C), and low-density lipoprotein cholesterol (LDL-C) were assayed with an automated biochemical analyzer (BS400, Mindary, Shenzhen, China). SOD, MDA, and FFAs
Table 1. Primers Used for Quantitative Real-Time PCR

\begin{tabular}{cll}
\hline \hline Gene & & \multicolumn{1}{c}{ Sequence 5' $\rightarrow 3^{\prime}$} \\
\hline \multirow{2}{*}{ GAPDH } & Forward & GCCAAAAGGGTCATCATCTC \\
& Reverse & GTAGAGGCAGGGATGATGTTC \\
Grp78 & Forward & CCTGCGTCGGTGTGTTCAA \\
& Reverse & ATCGCCAATCAGACGCTCC \\
Chop & Forward & GCCTTTCACCTTGGAGACGG \\
& Reverse & GGACGCAGGGTCAAGAGTAGTG \\
Caspase12 & Forward & ACAGCACATTCCTGGTGTTTATG \\
& Reverse & CAGACTCTGGCAGTTACGGTTG \\
Nlrp3 & Forward & AGCCTTCCAGGATCCTCTTC \\
& Reverse & CTGGGCAGCAGTTTCTTTC \\
\hline
\end{tabular}

were assayed in 10\% liver tissue homogenates using commercial kit following the manufacturer's instructions. An enzyme-linked immunosorbent assay kits specific for mouse IL-18 (Neobioscience, Shenzhen, China) was used following the manufacturer's instructions.

Histopathology Liver tissue samples were fixed for $24 \mathrm{~h}$ in $4 \%$ paraformaldehyde at $4{ }^{\circ} \mathrm{C}$ and then processed for paraffin embedding by standard methods. Embedded tissue blocks were serially sectioned at $4 \mu \mathrm{m}$ using a rotary microtome (Leica RM 2135, Meyer Instruments, Houston, TX, U.S.A.) and stained with hematoxylin and eosin (H\&E).

Quantitative Real-Time PCR (qPCR) Total RNA was extracted from hepatic tissue by the centrifugal column method following the manufacturer's protocol (Reagan, Beijing, China). The extracted RNA was reverse-transcribed to cDNA using all-in-one cDNA Synthesis SuperMix (Bimake, Houston, TX, U.S.A.) following the manufacturer's protocol. qPCR was performed with a Light Cycler system (Bio-Rad Laboratories, Inc., Hercules, CA, U.S.A.) using SYBR Green qPCR Master Mix (Biomarker, Houston, TX, U.S.A.). The forward and reverse primers are shown in Table 1 . The quantity of mRNA was normalized against an internal mouse $G A P D H$ standard.

Enzyme-Linked Immunosorbent Assay (ELISA) ELISA kits specific for mouse IL-18 (Neobioscience) was used following the manufacturer's instructions.

Western Blotting Liver tissue samples were homogenized in protein lysis buffer containing $1 \mathrm{~mm}$ phenylmethanesulfonyl fluoride and centrifuged at $12000 \mathrm{rpm}$ at $4^{\circ} \mathrm{C}$ for $15 \mathrm{~min}$. The supernatants were collected, and the protein concentration was determined by a bicinchoninic acid assay. Lysates containing equal amounts of protein were heated at $98^{\circ} \mathrm{C}$ in sodium dodecyl sulfate (SDS) sample buffer for $8 \mathrm{~min}$ and separated by polyacrylamide gel electrophoresis (PAGE). The proteins were transferred to polyvinylidene difluoride membranes (Bio-Rad), blocked with 5\% skimmed milk in Tris-buffered saline containing $0.5 \%$ Tween-20 for $1 \mathrm{~h}$ at room temperature, incubated overnight at $4^{\circ} \mathrm{C}$ with primary antibodies, and subsequently incubated with HRP-conjugated anti-rabbit IgG for $2 \mathrm{~h}$ at $37^{\circ} \mathrm{C}$. Specific bands were detected with enhanced chemiluminescence (ECL) using a Bio-Spectrum Gel Imaging System (Bio-Rad).

Statistical Analysis SPSS 20.0 software (IBM Corp., Armonk, NY, U.S.A.) was used for statistical analysis. Results were expressed as means \pm standard deviation (S.D.). The significance of between-group differences was determined by the 
independent $t$-test. Multiple comparisons were performed by one-way ANOVA. $p<0.05$ was considered statistically significant.

\section{RESULTS}

Rg1 Regulates Body Weight and Liver Wet Weight The body weight of mice in each group continued to increase during model establishment, and after 16 weeks the mice fed with a HFD were significantly heavier than controls (Fig. 1B). After 4 weeks of drug treatment, the body weight and liver wet weight of mice given Rg1 was significantly less than that of mice in the HFD group (Fig. 1C). The results indicated that Rg1 controlled the occurrence of obesity of HFD mice.

Rg1 Reduces the Level of Serum Transaminase and Blood Lipid in Mice Liver transaminases and blood lipid levels are key indicators of NAFLD. The HFD led to severe liver function damage as shown by increases of serum ALT and AST (Figs. 1D, E), TG, TC, HDL-C and LDL-C (Figs. $1 \mathrm{~F}-\mathrm{I})$. Serum ALT, AST, and TG were significantly decreased by Rg1 treatment, whereas decreases of serum TC, HDL-C, LDL-C after Rg1 treatment did not reach significance. These results suggested that $\mathrm{Rg} 1$ reduced liver inflammation and inhibited hepatic fat synthesis.

Rg1 Attenuates HFD-Induced Liver Hepatic Adipose Infiltration Histological evaluation of H\&E-stained sections of liver tissue revealed that the long-term HFDs led to diffuse fat degeneration, inflammation, balloon degeneration, and necrosis. Rg1 treatment significantly reduced in the signs of liver injury and decreased the extent of adipose infiltration (Fig. 1J).

Rg1 Increases Antioxidant Capacity and Promotes Lipid Peroxidation FFAs are the source of most triglycerides in the liver, which were higher in the HFD group than in the Rg1 groups, further confirming Rg1 accelerated TG metabolism (Fig. 2A). As FFAs are a source of oxidative stress in patients with fatty liver, SOD activity was assayed to evaluate oxidase activity and MDA was assayed as a marker of lipid peroxidation. SOD declined, and MDA increased in the HFD group compared with the CON group, and the extent of change was decreased by Rg1 (Figs. 2B, C). Rg1 thus increased antioxidant activity in this NAFLD model.

To investigate the cause of decreased FFAs in Rg1-treated HFD mice, the hepatic expression of genes involved in fatty acid $\beta$-oxidation was assayed. PPAR $\alpha$ expression, a key gene involved in $\beta$-oxidation of fatty acids, was decreased by the HFD, but the decrease was smaller in the mice given Rg1 (Fig. 2D). These results suggest that $\mathrm{Rg} 1$ promotes FFAs oxidation and inhibited TG synthesis upregulating PPAR $\alpha$ expression.

Rg1 Inhibits Expression of Genes Involved in ER Stress and Inflammasome Activation NAFLD is accompanied by ER stress that leads to apoptosis, inflammation and further aggravates hepatocellular damage. To investigate the mechanism of $\mathrm{Rg} 1$ reduction of inflammation, the expression of GRP78, Caspase 12 and CHOP, three genes that code for proteins associated with in ER stress, were assayed with qPCR and Western blotting. All three were increased in HFD mice, and the increase was inhibited by Rg1 treatment (Figs. 3A-D).

The NLRP3 inflammasome is an intracellular multiprotein complex involved in the production of mature IL- $1 \beta$ and IL-18. The expression of serum IL-18 concentrations, NLRP3, IL- $1 \beta$, and cleaved IL- $\beta$ proteins were all upregulated in the HFD group and the changes were all decreased by $\mathrm{Rg} 1$ treatment (Figs. 4A-C). The results indicated that the antiinflammatory activity of Rg1 included inhibition of ER stress and inflammasome activation.

\section{DISCUSSION}

NAFLD has been described as a component of metabolic syndrome along with obesity, diabetes and hypertension. ${ }^{25}$ ) It could be treated with metformin, statins, and fibrates, all of which have associated adverse reactions or contraindica-
A

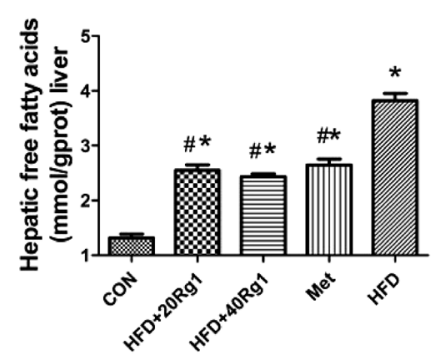

B

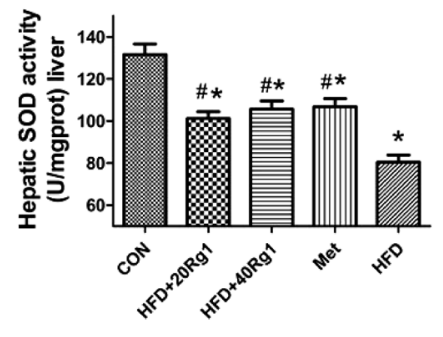

C

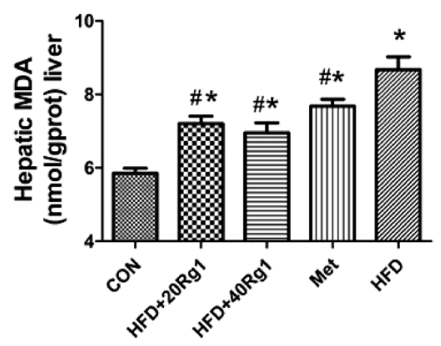

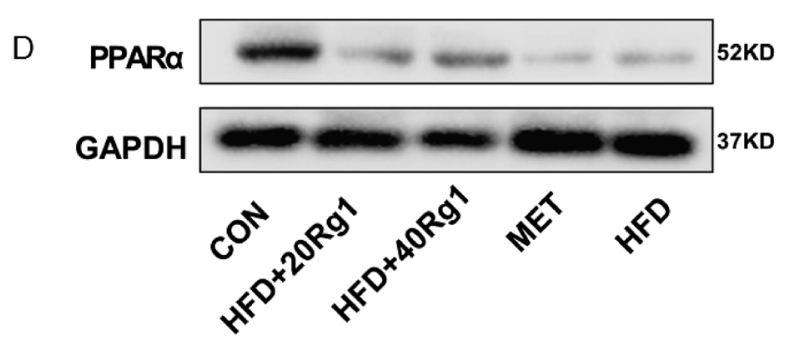

Fig. 2. Rg1 Protects Liver by Regulating Oxidative Stress and Ameliorating Lipid Peroxidation

The effects of Rg1 on free fatty acids (A), SOD activity (B) and MDA levels (C) in liver tissue. Data are means \pm standard error of the mean $(n=8)$. $* p<0.05$ versus CON, ${ }^{\#} p<0.05$ versus HFD. (C) Western blot assay of gene of PPAR $\alpha$ involved in $\beta$-oxidation. Representative images of at least three independent experiments are shown. CON, control group; HFD+20 Rg1, Rg1 low-dose group; HFD+40 Rg1, Rg1 high-dose group; Met, metformin group; HFD, high-fat diet group. 
A

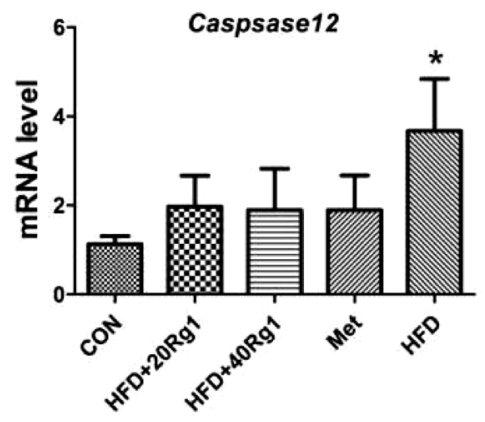

C

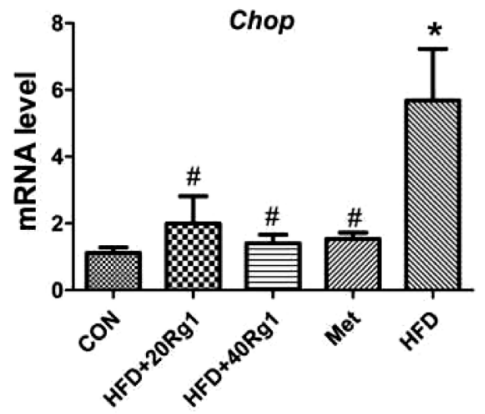

B
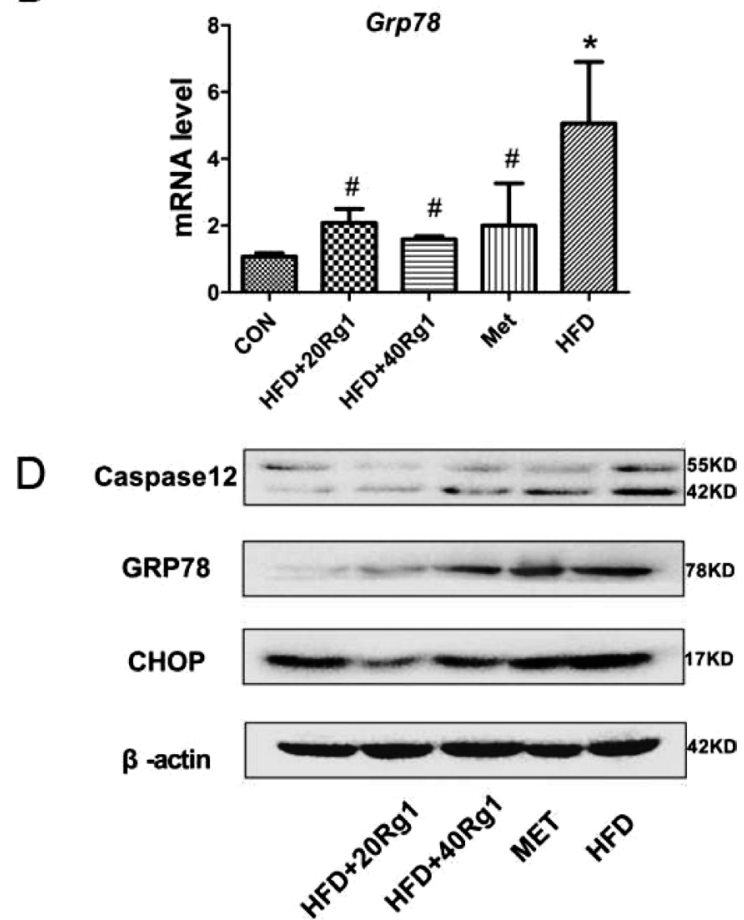

Fig. 3. Rg1 Alters Hepatic Expression of Genes Involved in ER Stress

Effects of Rg1 on the relative expression of Caspase 12 (A), Grp78 (B), and Chop (C) mRNA assayed with qPCR. Relative mRNA expression is shown as a ratio relative to GAPDH and reported as means \pm standard error of the mean $(n=5)$. ${ }^{*} p<0.05$ versus $\mathrm{CON}$, ${ }^{*} p<0.05$ versus HFD. (D) The effect of Rg1 on expression of ER stress proteins, including Caspase12, GRP78, and CHOP, assayed by Western blotting, with $\beta$-actin as a loading control. Representative images of at least three independent experiments are shown. CON, control group; HFD+20 Rg1, Rg1 low-dose group; HFD+40 Rg1, Rg1 high-dose group; Met, metformin group; HFD, high-fat diet group.

A

B
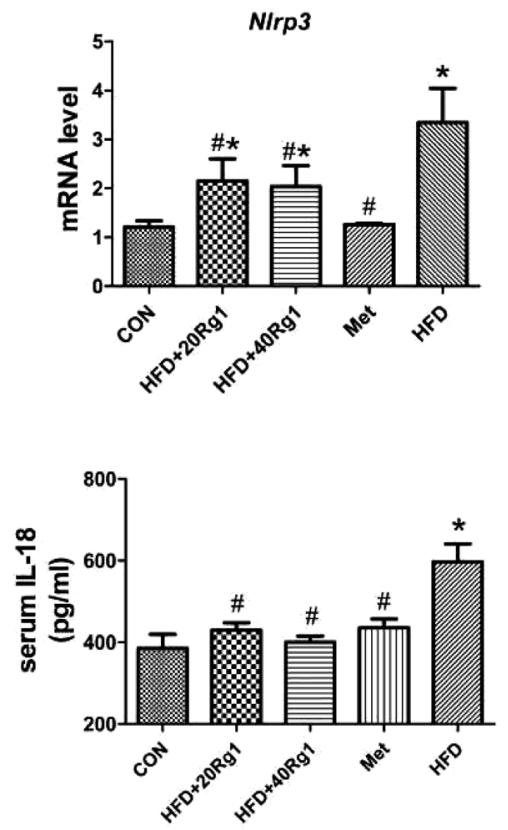

C

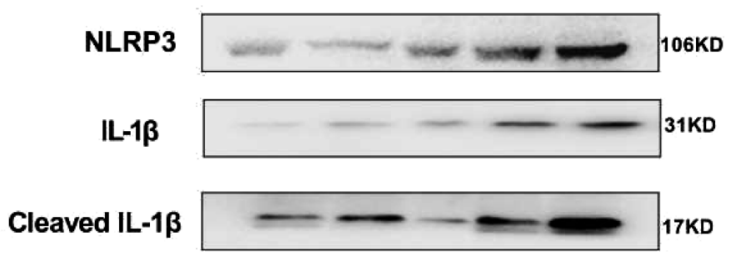

$\beta$-actin

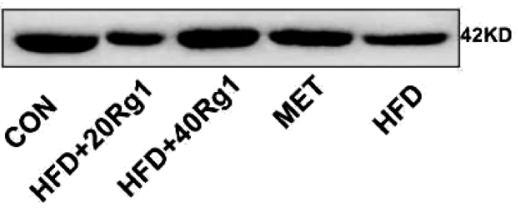

Fig. 4. Rg1 Ameliorates NAFLD Induced by High-Fat Diet Upregulation of Inflammasome Activation and Proinflammatory Cytokines

(A) Effects of Rg1 on the relative mRNA expression of Nlrp3 assayed with qPCR. (B) Serum IL-18 was assayed with ELISA. Data are means \pm standard error of the mean $(n=5)$. $* p<0.05$ versus CON, ${ }^{*} p<0.05$ versus HFD. (C) Western blot analysis of IL- $1 \beta$ and NLRP3 expression in mouse liver tissue. Representative images of at least three independent experiments are shown. CON, control group; HFD+20 Rg1, Rg1 low-dose group; HFD+40 Rg1, Rg1 high-dose group; Met, metformin group; HFD, high-fat diet group.

tions. ${ }^{26)}$ So far, no consensus has been reached on the most effective treatment. This study demonstrated the effects of Rg1 in a mouse NAFLD model induced by HFD. The protective effects of Rg1 involved a variety of signaling molecules and pathways.

The HFD-fed mice induced obesity, dyslipidemia, impaired 
liver function and hepatic steatosis, which are common risk factors for the development of NAFLD. Liver injury in the HFD group was accompanied by elevated serum transaminase, lipid and increased liver FFAs levels compared with the CON group. Rg1 treatment significantly reduced liver weight. It also decreased the elevation of serum ALT, AST, TG and the liver FFAs levels. Liver pathology was improved following Rg1 treatment. There was no statistically significant difference in the responses to the different doses of $\mathrm{Rg} 1$ over the time of treatment. The results showed that Rg1 had anti-inflammatory effects and improved lipid deposition.

The "two-hit" hypothesis in the progression of NAFLD involves the accumulation of triglycerides in hepatocytes, insulin resistance (IR), inflammation, ER stress, and oxidative stress. ${ }^{27)}$ The role of oxidative stress in NAFLD has been described, ${ }^{28)}$ and associated with increased FFAs levels that occur together with increased IR. The increased FFAs is linked to hepatic reactive oxygen species (ROS) production. This phenomenon could lead to the increased synthesis of MDA by upregulating microsomal lipid peroxidation and downregulating mitochondrial $\beta$-oxidation. ${ }^{29,30)}$ In this NAFLD model, the HFD produced a decrease of antioxidant capacity and generation of lipid peroxidation. Rg1 administration significantly increased antioxidant activity.

The liver is the primary site of lipid synthesis and metabolism as well as FFAs oxidation. The HFD feeding led to overload of FFAs and promotion of hepatocyte fat synthesis. Mitochondrial $\beta$-oxidation is the primary oxidative pathway for FFAs disposal. PPAR $\alpha$ expression, which regulates the expression of mitochondrial and peroxisomal $\beta$-oxidation enzymes, is inhibited with the development of IR. ${ }^{31)} \mathrm{Rg} 1$ upregulated PPAR $\alpha$ expression, which could result in an increase in the $\beta$-oxidation of fatty acids. The effect of $\operatorname{Rg} 1$ in preventing oxidative stress might result from reducing lipid peroxidation via $\operatorname{PPAR} \alpha$.

In response to nutrition overload, accumulation of unfolded protein in the ER increases ROS production and evokes ER stress, which involves in the development of NAFLD. ${ }^{32,33)}$ GPR78, CHOP, and Caspase 12, which are included in cell apoptosis, lipid metabolism, and tissue injury, are also markers of ER stress. Consequently, changes in expression of these proteins and the genes, imply involvement of increased ER stress during the development of diabetes. ${ }^{34)}$ In this NAFLD model, the elevated expression of GPR78, CHOP, and Caspase 12 in mouse liver was attenuated by $\operatorname{Rg} 1$ treatment. Activation of the unfolded protein response (UPR) is used as an indicator of ER stress. The increased expression of UPR signaling proteins may indicate the induction of ER stress in NAFLD. Thus, Rg1 treatment may have attenuated ER stress in the NAFLD tissue by reducing expression of those marker proteins. Severe or prolonged ER stress leads to inflammation and cell death. ${ }^{35)}$ We previously found that Rg1 inhibited inflammatory responses via modulation of the nuclear factor kappa-light-chain-enhancer of activated B cells (NF- $\kappa \mathrm{B})$ pathway and inflammasome activation in alcoholic hepatitis. ${ }^{23)}$ The NLRP3 inflammasome is a large intracellular multiprotein complex involved in mature IL- $1 \beta$ production and induces metabolic inflammation. ${ }^{36)}$ Expression of NLRP3 inflammasome, IL-18 and IL-1 $\beta$ are increased in the livers of nonalcoholic hepatic steatosis patients. ${ }^{37)}$ In this NAFLD model, NLRP3 inflammasome activity and expression of IL-18 and
IL- $1 \beta$ were increased by the HFD. Meanwhile, Rg1 prevented those responses. However, the association with ER stress remains to be determined in subsequent experiments. This further indicates that the anti-inflammatory effect of Rg1 may have been related to the inhibition of inflammasome activity.

Metformin was used as a positive control drug in the study. It can improve insulin sensitivity and treat NAFLD. Metformin significantly reduced body weight, serum transaminase and lipids in mice fed the HFD (Figs. 1A-H). It possessed somewhat similar effects as Rg1 in increasing the antioxidant enzyme activity, inhibiting ER stress and inflammation (Figs. 2-4). However, the effect of metformin on liver histology was not significant compared with Rg1 treatment. These results indicated that $\operatorname{Rg} 1$ has sufficient potential to treat NAFLD.

In conclusion, the present work firstly showed that Rg1 was effective in protecting against the development of NAFLD. It could not only improve hyperlipidemia and lipid peroxidation, but also reduce ER stress and inflammatory responses for liver function protection. Our experiments revealed preliminarily that multiple pathways were involved in its anti-NAFLD effect of Rg1. In the future, further experimental investigations are required to explore the additional mechanisms.

Acknowledgments This study was supported by Chongqing Research Program of Basic Research and Frontier Technology (Grant No. cstc2015jcyjBX0079).

Conflict of Interest The authors declare no conflict of interest.

Supplementary Materials The online version of this article contains supplementary materials.

\section{REFERENCES}

1) Buzzetti E, Pinzani M, Tsochatzis EA. The multiple-hit pathogenesis of non-alcoholic fatty liver disease (NAFLD). Metabolism, 65 , 1038-1048 (2016).

2) Sanyal AJ, Neuschwander-Tetri BA, Tonascia J. End points must be clinically meaningful for drug development in nonalcoholic fatty liver disease. Gastroenterology, 150, 11-13 (2016).

3) Younossi ZM, Koenig AB, Abdelatif D, Fazel Y, Henry L, Wymer M. Global epidemiology of nonalcoholic fatty liver disease-Metaanalytic assessment of prevalence,incidence, and outcomes. Hepatology, 64, 73-84 (2016).

4) Byrne CD, Targher G, European Association for the Study of the Liver (EASL), European Association for the Study of Diabetes (EASD), European Association for the Study of Obesity (EASO). EASL-EASD-EASO Clinical practice guidelines for the management of non-alcoholic fatty liver disease: Is universal screening appropriate? J. Hepatol., 64, 1388-1402 (2016).

5) Santhekadur PK, Kumar DP, Sanyal AJ. Preclinical models of nonalcoholic fatty liver disease. J. Hepatol., 68, 230-237 (2018).

6) Wattacheril J, Chalasani N. Nonalcoholic fatty liver disease (NAFLD): Is it really a serious condition? Hepatology, 56, 15801584 (2012).

7) Asrih M, Jornayvaz FR. Diets and nonalcoholic fatty liver disease: the good and the bad. Clin. Nutr., 33, 186-190 (2014).

8) Ozcan U, Cao Q, Yilmaz E, Lee AH, Iwakoshi NN, Ozdelen E, Tuncman G, Görgün C, Glimcher LH, Hotamisligil GS. Endoplasmic reticulum stress links obesity, insulin action, and type 2 diabetes. Science, 306, 457-461 (2004).

9) Cusi K. Role of insulin resistance and lipotoxicity in nonalcoholic 
steatohepatitis. Clin. Liver Dis., 13, 545-563 (2009).

10) Kersten S, Stienstra R. The role and regulation of the peroxisome proliferator activated receptor alpha in human liver. Biochimie, 136, 75-84 (2017).

11) Hotamisligil GS. Inflammation and endoplasmic reticulum stress in obesity and diabetes. Int. J. Obes., 32 (Suppl. 7), S52-S54 (2008).

12) Puri P, Mirshahi F, Cheung O, Natarajan R, Maher JW, Kellum JM, Sanyal AJ. Activation and dysregulation of the unfolded protein response in nonalcoholic fatty liver disease. Gastroenterology, 134, 568-576 (2008).

13) Toriguchi $\mathrm{K}$, Hatano $\mathrm{E}$, Tanabe $\mathrm{K}$, Takemoto $\mathrm{K}$, Nakamura $\mathrm{K}$, Koyama Y, Seo S, Taura K, Uemoto S. Attenuation of steatohepatitis, fibrosis, and carcinogenesis in mice fed a methionine-choline deficient diet by CCAAT/enhancer-binding protein homologous protein deficiency. J. Gastroenterol. Hepatol., 29, 1109-1118 (2014).

14) Malhi H, Kropp EM, Clavo VF, Kobrossi CR, Han J, Mauer AS, Yong J, Kaufman RJ. C/EBP Homologous protein-induced macrophage apoptosis protects mice from steatohepatitis. J. Biol. Chem., 288, 18624-18642 (2013).

15) Goodall JC, Wu C, Zhang Y, McNeill L, Ellis L, Saudek V, Gaston JS. Endoplasmic reticulum stress-induced transcription factor, CHOP, is crucial for dendritic cell IL-23 expression. Proc. Natl. Acad. Sci. U.S.A., 107, 17698-17703 (2010).

16) Lerner AG, Upton JP, Praveen PV, Ghosh R, Nakagawa Y, Igbaria A, Shen S, Nguyen V, Backes BJ, Heiman M, Heintz N, Greengard P, Hui S, Tang Q, Trusina A, Oakes SA, Papa FR. IRE1 $\alpha$ induces thioredoxin-interacting protein to activate the NLRP3 inflammasome and promote programmed cell death under irremediable ER stress. Cell Metab., 16, 250-264 (2012).

17) Vandanmagsar B, Youm YH, Ravussin A, Galgani JE, Stadler K, Mynatt RL, Ravussin E, Stephens JM, Dixit VD. The NLRP3 inflammasome instigates obesity-induced inflammation and insulin resistance. Nat. Med., 17, 179-188 (2011).

18) Zhang S, Zheng L, Dong D, Xu L, Yin L, Qi Y, Han X, Lin Y, Liu $\mathrm{K}$, Peng J. Effects of flavonoids from Rosa laevigata Michx fruit against high-fat diet-induced non-alcoholic fatty liver disease in rats. Food Chem., 141, 2108-2116 (2013).

19) Gao Y, Chu S, Zhang Z, Chen N. Hepataprotective effects of ginsenoside Rg1-A review. J. Ethnopharmacol., 206, 178-183 (2017).

20) Xu L, Chen WF, Wong MS. Ginsenoside Rg1 protects dopaminergic neurons in a rat model of Parkinson's disease through the IGF-I receptor signalling pathway. Br. J. Pharmacol., 158, 738-748 (2009).

21) Kim SJ, Yuan HD, Chung SH. Ginsenoside Rg1 suppresses hepatic glucose production via AMP-activated protein kinase in hepg2 cells. Biol. Pharm. Bull., 33, 325-328 (2010).

22) Zhao JQ, Shi Z, Liu S, Li J, Huang W. Ginsenosides Rg1 from Panax ginseng: A Potential Therapy for Acute Liver Failure Patients? Evid. Based Complement. Alternat. Med., 2014, 538059 (2014).

23) Li JJ, Yang C, Zhang S, Liu S, Zhao L, Luo H, Chen Y, Huang W. Ginsenoside Rg1 inhibits inflammatory responses via modulation of the nuclear factor- $\kappa \mathrm{B}$ pathway and inhibition of inflammasome activation in alcoholic hepatitis. Int. J. Mol. Med., 41, 899-907 (2018).

24) Kim SJ, Yuan HD, Chung SH. Ginsenoside Rg1 suppresses hepatic glucose production via AMP-activated protein kinase in HepG2 cells. Biol. Pharm. Bull., 33, 325-328 (2010).

25) Clark JM, Brancati FL, Diehl AM. Nonalcoholic fatty liver disease. Medicine, 347, 768-769 (2002).

26) Shin JH, Jung JH. Non-alcoholic fatty liver disease and flavonoids: Current perspectives. Clinics \& Research in Hepatology \& Gastroenterology, 41, 17-24 (2017).

27) Day CP, James OF. Steatohepatitis: A tale of two "hits"? Gastroenterology, 114, 842-845 (1998).

28) Koek GH, Liedorp PR, Bast A. The role of oxidative stress in nonalcoholic steatohepatitis. Clin. Chim. Acta, 412, 1297-1305 (2011).

29) Mitsuyoshi H, Itoh $Y$, Okanoue T. Role of oxidative stress in nonalcoholic steatohepatitis. Nihon Rinsho Japanese Journal of Clinical Medicine, 64, 1077-1082 (2006).

30) Xiao J, Ching YP, Liong EC, Nanji AA, Fung ML, Tipoe GL. Garlic-derived S-allylmercaptocysteine is a hepato-protective agent in non-alcoholic fatty liver disease in vivo animal model. Eur. J. Nutr., 52, 179-191 (2013).

31) Lee GH, Kim HK, Chae SW, Kim DS, Ha KC, Cuddy M, Kress C, Reed JC, Kim HR, Chae HJ. Bax inhibitor-1 regulates endoplasmic reticulum stress-associated reactive oxygen species and heme oxygenase-1 expression. J. Biol. Chem., 282, 21618-21628 (2007).

32) Seki S, Kitada T, Sakaguchi H. Clinicopathological significance of oxidative cellular damage in non-alcoholic fatty liver diseases. Hepatology Research the Official Journal of the Japan Society of Hepatology, 33, 132-134 (2005).

33) Ozcan U, Cao Q, Yilmaz E, Lee AH, Iwakoshi NN, Ozdelen E, Tuncman G, Görgün C, Glimcher LH, Hotamisligil GS. Endoplasmic reticulum stress links obesity, insulin action, and type 2 diabetes. Science, 306, 457-461 (2004).

34) Zhang Q, Li Y, Liang T, Lu X, Zhang C, Liu X, Jiang X, Martin RC, Cheng M, Cai L. ER stress and autophagy dysfunction contribute to fatty liver in diabetic mice. Int. J. Biol. Sci., 11, 559-568 (2015).

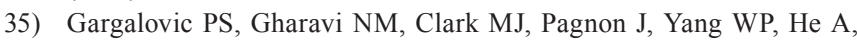
Truong A, Baruch-Oren T, Berliner JA, Kirchgessner TG, Lusis AJ. The unfolded protein response is an important regulator of inflammatory genes in endothelial cells. Arterioscler. Thromb. Vasc. Biol., 26, 2490-2496 (2006).

36) Wan XY, Xu CF, Yu CH, Li Y. Role of NLRP3 inflammasome in the progression of NAFLD to NASH. Chin. J. Gastroenterol. Hepatol., 2016, 6489012 (2016).

37) Wree $A$, Mcgeough MD, Peña CA, Schlattjan M, Li H, Inzaugarat ME, Messer K, Canbay A, Hoffman HM, Feldstein AE. NLRP3 inflammasome activation is required for fibrosis development in NAFLD. J. Mol. Med., 92, 1069-1082 (2014). 